\title{
ANÁLISIS CONGLOMERADO DE 15 CRUZAS DE CHILE PARA VARIABLES FENOLÓGICAS Y DE RENDIMIENTO ${ }^{1}$
}

\author{
Mirna Hernández-Pérez ${ }^{2}$, Alfonso López-Benítez ${ }^{2}$, Sergio Alfredo Rodríguez-Herrera ${ }^{2}$, \\ Fernando Borrego-Escalante ${ }^{2}$, Moisés Ramírez-Meraz ${ }^{3}$, Sandra Roxana López Benítez ${ }^{2}$
}

\section{RESUMEN}

Análisis conglomerado de $\mathbf{1 5}$ cruzas de chile para variables fenológicas y de rendimiento. El objetivo del presente trabajo fue determinar con el análisis cluster, las diferencias genotípicas y/o fenotípicas de quince cruzas directas de chile. Los progenitores fueron seis líneas de chile, tres eran jalapeños (4, 6 y 118), uno tipo húngaro amarillo (8), uno tipo ancho proveniente de Berentsen (16) y uno tipo mulato originario de CIAB (130). Los cruzamientos se realizaron en invernadero en la Universidad Autónoma Agraria Antonio Narro (UAAAN), en Saltillo, Coahuila, México. La evaluación se realizó en el campo experimental Las Huastecas del Instituto Nacional de Investigaciones Forestales, Agrícolas y Pecuarias (INIFAP), en Villa Cuauhtémoc, Tamaulipas, en el ciclo otoño-invierno de 2008. Se evaluaron las variables fenológicas: días a floración y días al primer corte; y las variables de rendimiento: número de promedio de frutos por plantas), peso promedio por fruto $\mathrm{y}$ toneladas por hectárea. Hubo relación positiva entre días al primer corte y días a floración y una relación negativa entre frutos por planta y peso promedio por fruto. El componente principal fue el de corte y el conglomerado resultó en tres grupos, el primero constituido por las cruzas 4x6, 4x8, 4x16, $4 \times 130,118 \times 130,8 \times 118,4 \times 118,6 \times 118,6 \times 16,6 \times 130,8 \times 16$, $8 \times 130$ y $16 \times 118$, el segundo por la cruza $16 \times 130$ y el tercero por $6 \times 8$. Esta agrupación puede deberse tanto al origen de los progenitores como al rendimiento obtenido.

Palabras clave: Capsicum annuum L., análisis cluster, caracteres fenológicos, componentes de rendimiento.

\begin{abstract}
Cluster analysis for phenological and yield variables in 15 crosses of pepper. The objective of this work was to determine the genotypic or phenotypics differences among 15 single crosses of hot pepper utilizing the cluster analysis. The parents of these crosses were six lines of pepper, three of them were jalapeño type (4, 6 y 118), one was Hungarian yellow wax type (8), one was a broad type (16) and one was mulatto (type 130). Crosses were conducted in a greenhouse at the Universidad Autónoma Agraria Antonio Narro (UAAAN), Saltillo, Coahuila, México. The field assessment was conducted at the Experimental Field Las Huastecas of the National Institute for Forest, Agricultural and Animal Research (INIFAP), at Villa Cuauhtémoc, Tamaulipas, in the autumn-winter cycle of 2008. The phenological variables evaluated were days to flowering and days to first harvest. Yield variables evaluated were the number of fruits per plant, fruit average weight, and yield ( $\mathrm{t} / \mathrm{ha}$ ). There was a positive relationship between days at the first harvest and days at flowering, and a negative relationship between fruits per plant and fruit average weight. The main component was days to first harvest. The cluster analysis indicated three groups, the first one constituted by crosses $4 \times 6,4 \times 8,4 \times 16,4 \times 130$, $118 \times 130,8 \times 118,4 \times 118,6 \times 118,6 \times 16,6 \times 130,8 \times 16,8 \times 130 y$ $16 \times 118$, the second by the $16 \times 130$ cross, and the third by the cross $6 \times 8$. This grouping may be a consequence of both the origin of the parents and the yield obtained.
\end{abstract}

Key words: Capsicum annuum L., cluster analysis, phenological traits, yield components.

\footnotetext{
1 Recibido: 22 de noviembre, 2009. Aceptado: 16 de mayo, 2011. Forma parte de una tesis doctoral, titulada: Efectos genéticos en la resistencia a la marchitez causada por Phytophthora capsici en Chile (Capsicum annиum) y en caracteres agronómicos importantes en rendimiento.

2 Departamento de Fitomejoramiento, Universidad Autónoma Agraria Antonio Narro (UAAAN). Dir. Hacienda de Enmedio, manzana 14, lote 32, casa B. Toluca, Edo. de México. C.P. 50210. hepemi@hotmail.com, alobe42@hotmail.com (correspondencia), serroh90@hotmail.com, fborregoe@hotmail.com

3 Investigador del Campo Experimental Las Huastecas. Instituto Nacional de Investigaciones Forestales, Agricolas y Pecuarias (INIFAP) Villa Cuauhtémoc, Tamaulipas.mramirezmz@yahoo.com.mx
} 


\section{INTRODUCCIÓN}

El chile (Capsicum annumm L.), es una hortaliza y condimento muy cotizado y conocido mundialmente (Laborde y Pozo 1984). Su aceptación es evidente por su consumo a nivel mundial. La especie $C$. апnиит var. Anпиит que es originaria de México, es la que presenta mayor variabilidad de formas cultivadas, se encuentra distribuida en todo el mundo y tiene amplia diversidad de tamaños, formas, colores, rango de maduración y grado de pungencia (Valadez 1998).

En México, el chile es una de las especies hortícolas más importantes debido al valor de su producción y a la alta demanda de mano de obra que genera. Se cultiva casi en todos los estados de la república, desde altitudes a nivel del mar hasta los $2500 \mathrm{msnm}$ y por ser su centro de origen, se han generado una gran diversidad de tipos y variedades, lo cual constituye un valioso recurso para su mejoramiento genético (Laborde y Pozo 1984).

La superficie sembrada en el territorio mexicano fluctúa alrededor de las 180 mil hectáreas, de las cuales más del $90 \%$ cuenta con sistemas de riego. El rendimiento presenta grandes diferencias entre la siembra con riego y la de temporal, desde $38 \mathrm{t} / \mathrm{ha}$ en el cultivo de chile bell en condiciones de riego, hasta 0,14 t/ha en chile piquín de temporal. Regularmente el rendimiento bajo sistema de riego es por lo menos el doble del obtenido en condiciones de secano (FAOSTAT 2007).

El cluster análisis, es un conjunto de técnicas que se utilizan para clasificar los objetos o casos en grupos relativamente homogéneos llamados conglomerados (Manly 1994). Los objetos en cada grupo (conglomerado) tienden a ser similares entre sí con alta homogeneidad interna, dentro del cluster y diferentes a los objetos de los otros grupos, alta heterogeneidad externa, ente clusters con respecto a algún criterio de selección predeterminado. De este modo, si la clasificación es un éxito, los objetos dentro del cluster estarán muy cercanos unos de otros en la representación geométrica, y los clusters diferentes estarán muy apartados. Este análisis se conoce también como análisis de clasificación o taxonomía numérica.

El conglomerado tiene como propósito esencial, agrupar aquellos objetos que reúnan características idénticas, es decir, se convierte así en una técnica de análisis exploratorio diseñada para revelar las agrupaciones naturales dentro de una colección de datos. Este análisis no hace ninguna distinción entre variables dependientes (VD) y variables independientes (VI) sino que calcula las relaciones interdependientes de todo el conjunto de variables (Anderberg 1973).

La mayoría de los métodos de agrupación son basados en un proceso de aglomeración o división son, heurísticos y fundamentados en algoritmos (Johnson y Wichern 1988). Otros métodos consisten en definir $\mathrm{K}$ grupos aleatorios y entonces se mueven los objetos entre estos para reducir la variación dentro de ellos y maximizar sus diferencias (Ward 1963). Llaurado y Moreno (1993) mediante la metodología de la clasificación jerárquica de Cornelius et al. (1993) estudiaron poblaciones de maíz de polinización abierta, utilizando como criterios de clasificación características morfológicas y las clasificaron en ocho grupos. Zamora (2001) trabajando con este método, propone definir un tercer grupo de triticales "intermedio-invernal" con características propias que lo diferencian de los primaverales, facultativos e invernales. Montesinos (2003) organiza 45 genotipos de melón (Cucumis melo L.) en cuatro grupos de acuerdo a características fisiotécnicas y de tolerancia a la cenicilla polvorienta (Erysiphe cichoracearum y Sphaerotheca fuliginea).

Las medidas de proximidad, similitud o semejanza miden el grado de ésta entre dos objetos. De esta manera, cuanto mayor (respuesta menor) es su valor, mayor es el grado de similaridad existente entre ellos, y métodos de clasificación tenderán a ponerlos en el mismo grupo.

En un trabajo con once morfotipos de chile, la mayoría de ellos silvestres, se encontró que en el análisis de componentes principales (ACP) indicaba que con los primeros cuatro se explicó el $88 \%$ de la variación morfológica total (Castañón et al. 2008). Este análisis (ACP) agrupó a los morfotipos silvestres en un grupo y a los comerciales en otro. El análisis de conglomerados (AC) permitió clasificar a los morfotipos en grupos contrastantes; ese agrupamiento se dio principalmente por la procedencia, cultivado o silvestre, a cada morfotipo. Así, los morfotipos comerciales formaron un grupo aparte respecto a los silvestres. Es decir, en general, los morfotipos se agruparon como en el ACP. En ambos análisis, las variables que más participaron en la agrupación de los morfotipos fueron el color de las anteras, la posición de la flor y el ancho del fruto.

Con el fin de conocer la variabilidad cualitativa y cuantitativa de 111 poblaciones del género Capsicum, 
a cargo de CORPOICA en el Centro de Investigación "La Selva" (Medina et al. 2006), analizaron las especies $C$. annuиm, $C$. baccatum, $C$. chinense, $C$. frutescens, $C$. pubescens y C. spp. (silvestres). El valor de la variabilidad cualitativa $(95,7 \%)$, considerado en forma conjunta con el valor máximo de disimilitud $(52 \%)$, señala que a nivel de accesiones individuales hay un nuevo ordenamiento o arreglo de los atributos cualitativos, lo que permite seleccionar materiales con las combinaciones deseadas de esos atributos.

El objetivo del presente trabajo fue determinar con el análisis cluster, las diferencias genotípicas y/o fenotípicas de quince cruzas directas de chile.

\section{MATERIALES Y MÉTODOS}

Área de estudio y material genético: Las cruzas se realizaron bajo un diseño dialélico en las instalaciones de la Universidad Autónoma Agraria Antonio Narro (UAAAN), ubicada al sur de la Ciudad de Saltillo, Coahuila, México. Los materiales genéticos utilizados en el cruzamiento fueron seis líneas de chile de diferentes tipos (Cuadro 1). La evaluación de las quince cruzas simples resultantes (Cuadro 2), se evaluaron en el Campo Experimental Las Huastecas (CEHUAS), del Instituto Nacional de Investigaciones Forestales, Agrícolas y Pecuarias (INIFAP), localizado en el km 55 de la carretera Tampico-Mante, al nororiente de la República Mexicana. Se establecieron las quince cruzas resultantes del dialélico (Cuadro 1) en el ciclo primavera-verano del 2008 bajo un diseño experimental de bloques completos al azar con cuatro repeticiones. De cada cruza se establecieron dos surcos de $2,5 \mathrm{~m}$ de longitud, una separación entre estos de $0,9 \mathrm{~m}$ y 0,3 $\mathrm{m}$ entre plantas para una densidad de 32000 plantas por hectárea.

El manejo del experimento en general fue de acuerdo con las recomendaciones del mismo campo experimental.

\section{Variables evaluadas}

Días a floración (DAF): la estimación se hizo contando el número de días transcurridos desde el trasplante hasta la floración del 50\% del genotipo evaluado.

Días a cosecha (DAC): se contó el número de días a partir de la fecha del trasplante, al primer corte de
Cuadro 1. Líneas de chile utilizadas como progenitores en el cruzamiento dialélico. Universidad Autónoma Agraria Antonio Narro. Saltillo, Coahuila, México. Otoño 2007.

\begin{tabular}{lll}
\hline Líneas & Descripción & Origen \\
\hline 4 & Tipo Jalapeño & UAAAN $^{1}$ \\
6 & Tipo Jalapeño & UAAAN \\
8 & Tipo Húngaro Amarillo & UAAAN \\
16 & Tipo Ancho & C. S. B.Berensen \\
\\
118 & Tipo Jalapeño & R. B. T. \\
130 & Tipo Mulato & CIAB $^{4}$ \\
\hline
\end{tabular}

${ }^{1}$ UAAAN = Universidad Autónoma Agraria Antonio Narro. Saltillo, Coahuila. México. Otoño 2007.

${ }^{2}$ C. S. B. = Compañía de semillas Berensen.

${ }^{3}$ R. B. T. = Río Bravo, Tamaulipas, México.

${ }^{4} \mathrm{CIAB}=$ Centro de Investigaciones Agrícolas del Bajío. Celaya; Guanajuato. México.

Cuadro 2. Cruzas $\mathrm{F}_{1}$ de chile, evaluadas para días a floración (DAF), días a cosecha (DAC), número de frutos (NTF) y peso promedio de frutos (PPF). Campo Experimental Las Huastecas (CEHUAS), México. Primavera-verano 2008.

\begin{tabular}{ccc}
\hline & Cruzas & Descripción \\
\hline 1 & $4 \times 6$ & Cruza simple \\
2 & $4 \times 8$ & Cruza simple \\
3 & $4 \times 16$ & Cruza simple \\
4 & $4 \times 118$ & Cruza simple \\
5 & $4 \times 130$ & Cruza simple \\
6 & $6 \times 8$ & Cruza simple \\
7 & $6 \times 16$ & Cruza simple \\
8 & $6 \times 118$ & Cruza simple \\
9 & $6 \times 130$ & Cruza simple \\
10 & $8 \times 16$ & Cruza simple \\
11 & $8 \times 118$ & Cruza simple \\
12 & $8 \times 130$ & Cruza simple \\
13 & $16 \times 118$ & Cruza simple \\
14 & $16 \times 130$ & Cruza simple \\
15 & $118 \times 130$ & Cruza simple \\
\hline
\end{tabular}


todos y cada uno de los genotipos, y así se determinó su precocidad.

Número promedio de frutos (NTF): se cortaron todos los frutos de todas las plantas de cada genotipo y el total se dividió entre el número de plantas cosechadas.

Peso promedio de frutos (PPF): fueron pesados y divididos entre el número de frutos.

Rendimiento: se calculó el rendimiento obtenido de ocho plantas de un genotipo en $2,25 \mathrm{~m}^{2}$ y mediante el cálculo una regla de tres simple se estimó el rendimiento por hectárea (t/ha).

\section{Análisis estadísticos}

Se llevó a cabo un análisis de componentes principales (ACP) con las variables evaluadas y estandarizados a $\mu=0$ y $\sigma 2=1$. Este análisis de componentes principales se realizó con la matriz de correlaciones, con la finalidad que las variables involucradas tuvieran la misma importancia (Manly 1994, Crossa et al. 1995). La significancia de los eigenvalores y eigenvectores se determinó con la regla de Kaiser (1960). También se hizo un análisis de conglomerados jerárquico (AC) mediante el método de ligamiento promedio (UPGMA) con datos estandarizados de las variables a $\mu=0$ y $\sigma 2=1$. La razón para usar datos estandarizados es que las unidades en que las mismas fueron medidas, pueden afectar arbitrariamente la similaridad entre las entidades evaluadas. Además, la estandarización hace que los atributos medidos contribuyan de forma más equilibrada al cálculo de las similaridades (Lévy y Varela 2003). La medida de disimilitud fue la distancia euclidiana. Los dos procedimientos estadísticos se realizaron en SAS (2002).

\section{RESULTADOS Y DISCUSIÓN}

\section{Correlaciones}

Se presentó una correlación altamente significativa y positiva entre las variables de días a floración (DAF) y días a cosecha (DAC) (Cuadro 3), lo que nos enfatiza que a mayor DAC, más incremento habrá en DAF y viceversa, esto nos puede indicar tanto precocidad como materiales tardíos.

De igual forma se observa una correlación altamente significativa pero negativa entre las variables Ftos/pta y PPF (Cuadro 3), lo que nos indica que al momento de incrementarse la cantidad de frutos por planta disminuye de manera proporcional su peso promedio por fruto, y por el contrario, si se disminuye la cantidad de estos por planta, mayor será el peso promedio.

\section{Eigenvalores}

Se obtuvieron valores de varianza que explican cada componente como porcentaje de la varianza total. El primero explicó el 91,87\% del total (Cuadro 4), por lo que este es el componente principal (Walker 1980).

\section{Contribución relativa}

Se encontraron valores significativos en el componente uno, en las características de corte (DAF y DAC), por lo que se puede llamar de corte. Para el componente dos no se encontraron valores altos en ninguna de las características; sin embargo, el valor más alto lo tiene $\mathrm{t} / \mathrm{ha}$, por lo cual puede ser llamado componente de rendimiento (Cuadro 5).

Cuadro 3. Correlaciones entre variables de fenológicas y de rendimiento de quince cruzas de chile (Capsicum annuum L) evaluadas en el Campo Experimental Las Huastecas (CEHUAS). Instituto Nacional de Investigaciones Forestales, Agrícolas y Pecuarias (INIFAP), Villa Cuauhtémoc, Tamaulipas. Ciclo otoño-invierno de 2008.

\begin{tabular}{lccccc}
\hline & Días a floración & $\begin{array}{c}\text { Días al primer } \\
\text { corte }\end{array}$ & $\begin{array}{c}\text { Número total de } \\
\text { frutos }\end{array}$ & $\begin{array}{c}\text { Peso promedio } \\
\text { por fruto }\end{array}$ & $\begin{array}{c}\text { Rendimiento } \\
(\mathbf{t} / \mathbf{h a})\end{array}$ \\
\hline Días a floración & 1 & 0,837 & 0,381 & $-0,566$ & 0,103 \\
Días al primer corte & & 1 & 0,224 & $-0,459$ & $-0,108$ \\
Número total de frutos & & & 1 & $-0,646$ & 0,263 \\
Peso promedio por fruto & & & & 1 & 0,184 \\
Rendimiento & & & & 1 \\
\hline
\end{tabular}


Cuadro 4. Análisis de componentes principales (Eingenvalores) entre variables en quince cruzas de chile (Capsicum annuum L) evaluadas en el Campo Experimental Las Huastecas (CEHUAS). Instituto Nacional de Investigaciones Forestales, Agrícolas y Pecuarias (INIFAP), Villa Cuauhtémoc, Tamaulipas, México. Ciclo otoño-invierno de 2008.

\begin{tabular}{lcccc}
\hline Eigenvalor & $\begin{array}{c}\text { Varianza } \\
\text { total }(\boldsymbol{\%})\end{array}$ & $\begin{array}{c}\text { Eigenvalor } \\
\text { acumulado }\end{array}$ & $\begin{array}{c}\text { Acumulado } \\
(\boldsymbol{\%})\end{array}$ \\
\hline $1^{*}$ & 1,8374 & 91,87 & 1,84 & 91,87 \\
$2^{* *}$ & 0,1626 & 8,13 & 2,00 & 100,00 \\
\hline
\end{tabular}

*Componente $1=$ Días al primer corte.

***Componente $2=$ Rendimiento.

En la Figura 1, en el fenograma para las quince cruzas, con distancia euclidiana y no estandarizado, podemos observar tres grupos:

En el primer grupo están la mayoría de las cruzas (13), los cuales son: 4x6, 4x8, 4x16, 4x130, $118 \times 130,8 \times 118,4 \times 118,6 \times 118,6 \times 16,6 \times 130,8 \times 16$, $8 \times 130$ y $16 \times 118$. En el segundo grupo está la cruza 16x130, y en el tercero la 6x8. Esta agrupación puede deberse a que todos los materiales pertenecen a la misma especie de Capsicum y a que la mayoría de
Cuadro 5. Contribución relativa de cada variable en los componentes principales en quince cruzas de chile (Capsicum annum L) evaluadas en el Campo Experimental Las Huastecas (CEHUAS). Instituto Nacional de Investigaciones Forestales, Agrícolas y Pecuarias (INIFAP), Villa Cuauhtémoc, Tamaulipas, México. Ciclo otoño-invierno de 2008.

\begin{tabular}{lcc}
\hline & Corte & Rendimiento \\
\hline Días a floración & 0,958478 & 0,285168 \\
Días al primer corte & 0,958478 & $-0,285168$ \\
Número total de frutos & 0,315281 & 0,275883 \\
Peso promedio por fruto & $-0,535222$ & $-0,186951$ \\
Rendimiento & $-0,002759$ & 0,369951 \\
\hline
\end{tabular}

ellos fueron obtenidos originalmente en mercados. Por el contrario, Medina et al. (2006) estudiaron diferentes poblaciones de $C$. annuиm, $C$. baccatum, $C$. chinense, $C$. frutescens, $C$. pubescens respecto de 47 atributos cualitativos y 22 cuantitativos. Los atributos cualitativos mostraron una similaridad mínima de $48 \%$ entre poblaciones, indicando arreglos genéticos particulares dentro de poblaciones individuales. Los atributos cuantitativos, mostraron amplia variabilidad, particularmente los relacionados con el fruto.

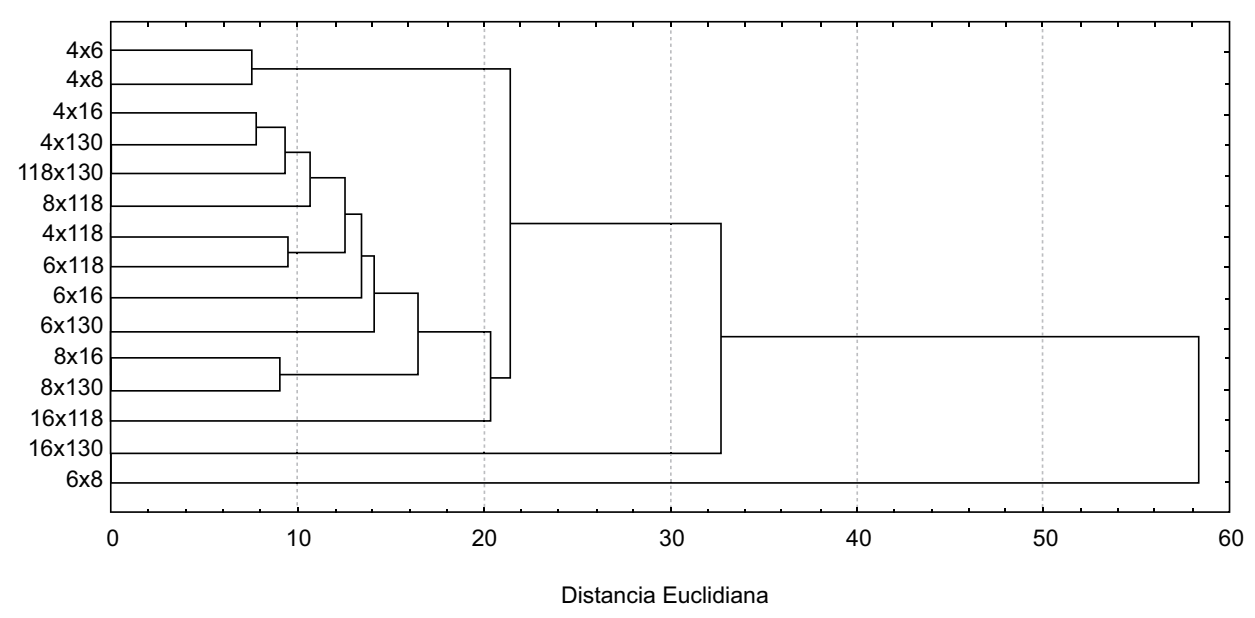

Figura 1. Fenograma del análisis de conglomerados con distancia euclidiana, estandarizado, efectuado a las quince cruzas de chile (Capsicum annuum L) evaluadas en el Campo Experimental Las Huastecas (CEHUAS). Instituto Nacional de Investigaciones Forestales, Agrícolas y Pecuarias (INIFAP), Villa Cuauhtémoc, Tamaulipas, México. Ciclo otoño-invierno de 2008. 
Las especies $C$. baccatum y $C$. pubescens formaron conglomerados únicos, lo cual refleja la significativa separación taxonómica de estas dos entidades biológicas con relación a los otros taxa domesticados del complejo $C$. annuum-chinense frutescens, entre los cuales se presentó intercalamiento en los conglomerados de los fenogramas. Aparentemente, los silvestres estudiados están relacionados con $C$. annиит dado su agrupamiento por atributos cualitativos, lo cual también se presentó a nivel de las variables cuantitativas. Se apreciaron agrupamientos de cada uno de los diferentes taxa en el fenograma cuantitativo con intercalamientos interespecíficos, lo cual indica transposición de los hábitat en los cuales se encuentran dichos materiales. En los dendrogramas cuantitativo y cualitativo, las especies silvestres estuvieron ubicadas cerca de demes de C. аnпиит, lo cual señala afinidades entre este taxón cultivado y las poblaciones silvestres evaluadas. Finalmente, se constató una amplia variabilidad morfológica cualitativa y cuantitativa que indica un potencial grande en la utilización de las poblaciones estudiadas para apoyar programas de mejoramiento del género Capsicum.

El componente principal detectado fue el de corte, con las variables: días a floración y días a cosecha con un $91,57 \%$ del total de la varianza. El otro $8,13 \%$ lo conformó el componente de rendimiento pero no fue significativo.

\section{LITERATURA CITADA}

Anderberg, MR. 1973. Cluster analysis for applications. Academia Press, New York. 359 p.

Castañón, NG; Latournerie, ML; Mendoza, EM; Vargas, LA; Cárdenas, MH. 2008. Colección y caracterización de chile (Capsicum spp) en Tabasco, México. Revista Pyton 77:189-202.

Cornelius, PL; Van Sanford, DA; Seydsard, MS. 1993. Clustering cultivars into groups without rank change interactions. Crop Science 33:1193-1200.

Crossa, J; DeLacy, IH; Taba, S. 1995. The use of multivariate methods in developing a core collection. In Hodgkin, T; Brown, AH; van Hintum, Th. JL; Morales, EAV. eds. Core Collections of Plant Genetic Resources. Wiley and Sons, IPGRI and Sayce Publishing. p. 77-92.
FAOSTAT. 2007. Organización de las naciones unidas para la agricultura y la alimentación (en línea). Consultado: 09 oct. 2009. Disponible en: http://faostat.fao. org/site/339/default.aspx

Johnson, RA; Wichern, DW. 1988. Applied multivariety statistical analysis. 2 ed. Prentice Hall. New Jersey. USA. 607 p.

Kaiser, HF. 1960. The application of electronic computers to factor analysis. Educational and Phsychological Measurement 20:141-151.

Laborde, CA; Pozo, CO. 1984. Presente y pasado del chile en México. SARH-INIA. Publicación Especial No. $85.80 \mathrm{p}$.

Lévy, MJP; Varela, M. 2003. Análisis multivariable para las ciencias sociales. Pearson Educación, S. A., Madrid. $896 \mathrm{p}$.

Llaurado, M; Moreno, G. 1993. Classification of northen spanish populations of maizeby methods of numerical taxonomy. I. Morphological traits. Maydica:15-21.

Manly, BFJ. 1994. Multivariate statistical methods. Chapman \& Hall. 215 p.

Montesinos, CA. 2003. Selección de progenies de melón (Cucumis melo L.) para alta eficiencia fisiotécnica y tolerantes a la cenicilla polvorienta. Tesis Maestría. Universidad Autónoma Agraria Antonio Narro. Buenavista, Saltillo, Coahuila, México. 15 p.

Manly, BFJ. 1994. Multivariate statistical methods. Chapman \& Hall. 215 p.

Medina, CI; Lobo, M; Farley, GA. 2006. Variabilidad fenotípica en poblaciones de ají y pimentón de la colección colombiana del género Capsicum. Revista Corpoica - Ciencia y Tecnología Agropecuaria 7(2):25-39.

SAS Institute Inc. 2002. SAS/STAT User's Guide. Versión 9. SAS institute Inc. Cary, NC. 228 p.

Valadez, LA. 1998. Producción de hortalizas. 1ra. ed. 8va. Reimpresión. Ed. Limusa. México. 298 p.

Ward, JH. 1963. Hierarchical grouping to optimize an objective function. J. Am. Stat. Assoc. 58:236-244.

Walker, ME. 1980. Métodos multivariados para el análisis de datos. Seminarios Técnicos. Vol. 3. 45 p.

Zamora, VVM. 2001. Agrupamiento, selección y estabilidad de la producción y calidad de triticales forrajeros (X. Tritivosecale Wittmac). Tesis Doctoral Fitomejoramiento. Universidad Autónoma Agraria Antonio Narro. Saltillo, Coahuila. México. 148 p. 